\title{
Contracting HIV or Contracting SAR-CoV-2 (COVID- 19) in Pregnancy? Balancing the Risks and Benefits
}

\author{
Dvora Joseph Davey ${ }^{1,2} \cdot$ Linda-Gail Bekker $^{3} \cdot$ Thomas J. Coates $^{1,4} \cdot$ Landon Myer $^{2}$
}

Published online: 13 April 2020

(c) Springer Science+Business Media, LLC, part of Springer Nature 2020

Scientists, governments and ethics committees must weigh the risks and benefits of continuing with HIV prevention studies, including HIV pre-exposure prophylaxis (PrEP) provision to high risk populations such as pregnant and breastfeeding women. The risk of HIV acquisition to women and infants is high and will be higher during the SARS-CoV-2 pandemic $[1,2]$. We argue that the benefit outweighs the risk in this population and advocate for continued PrEP enrolment, drug provision and adherence counselling in HIVuninfected pregnant and breastfeeding women at high risk of HIV acquisition in South Africa. South Africa has the largest coronavirus epidemic in Africa, with over 1650 cases in all nine Provinces in its early days [3]. The South African Department of Health reported eleven deaths of individuals infected with coronavirus this early date [4], and South African healthcare facilities are getting prepared for the next stage in the epidemic, wherein people with severe coronavirus require intensive care and ventilation [5].

On top of a potentially devastating COVID-19 epidemic, South Africa has the largest HIV epidemic in the world, with 7.7 million people living with HIV (PLHIV) and approximately 3 million PLHIV not on treatment [6]. South Africa's response to the HIV epidemic has worked, with $91 \%$ of people living with HIV (PLHIV) knowing their HIV status, 68\% on antiretroviral treatment (ART), and $83 \%$ achieving viral

Dvora Joseph Davey

dvoradavey@g.ucla.edu

1 Department of Epidemiology, Fielding School of Public Health, University of California Los Angeles, Los Angeles, USA

2 Division of Epidemiology \& Biostatistics, School of Public Health and Family Medicine, University of Cape Town, Cape Town, South Africa

3 Desmond Tutu HIV Centre, University of Cape Town, Cape Town, South Africa

4 David Geffen School of Medicine, University of California Los Angeles, Los Angeles, USA suppression [7]. HIV testing and treatment are standard of care in antenatal clinics. In 2019, the government rolled out PrEP for HIV in public health facilities [8]. Life-expectancy in South Africa has increased from 52 years in males and 56 years in females in 2006 to 61 and 67 years in 2018, respectively [9]. Vertical transmission of HIV has reduced dramatically from $3.5 \%$ in 2010 to $0.9 \%$ in 2018 [10].

President Cyril Ramaphosa of South Africa, instituted a national 21-day lockdown on Thursday 26 March, 2020 [3], with the objective of preventing "a human catastrophe of enormous proportions" [3]. As a result, the South African Department of Health closed down all non-essential health services and elective surgeries to focus on coronavirus diagnostics and care. Some ethics committees have followed suit and have shut down HIV research studies. For example, University of Cape Town's Human Research Ethics Committee (HREC) de-escalated or suspended research activities, prioritizing limiting infections and protect research participants, staff and students [11].

We are conducting studies in Gugulethu, one of the townships around Cape Town, to determine optimal strategies for providing PrEP to pregnant HIV-uninfected women. We stopped enrolling new participants in our PrEP in pregnancy and postpartum study (PrEP-PP) in agreement with the University of Cape Town's Human Research Ethics Committee (HREC) decision. We decided to remain open to PrEP retention visits, when the participant was not able to attend prior to site closing. However, we know that during emergencies and disasters like coronavirus, in which people are told to stay home, sex will continue or increase, and risks of intimate partner violence and rape increase [12, 13].

The South African Department of Health released guidelines on March 26, 2020 stating that "While health workers will continue with efforts to initiate and retain HIV positive individuals on ARV treatment during the National Lockdown, every effort will be made to also continue to reduce the number of new infections." In this note, we weigh the risks of continuation of PrEP provision for pregnant and 
breastfeeding women compared to the benefits of continuation during the COVID-19 pandemic.

\section{Risk of Continuation: Increased Coronavirus Exposure and Potential Infection of Participants and Staff}

Encouraging women to come into a crowded health facility could expose them to coronavirus if precautions are not taken. Even where precautions and screening are taking place, asymptomatic carriers can infect pregnant women or their infants with coronavirus [5]. Further, research staff and healthcare providers are at increased risk if they do not heed infection prevention precautions including the use of personal protective equipment like masks and gloves. Nurses who draw blood or test for HIV, will be at highest risk because of the proximity to patients and potential for patients who cough or sneeze on them during blood draws and HIV testing.

The risk would be greatest for women with other health conditions including tuberculosis, hypertension and diabetes. For women who are no longer coming to the clinic for antenatal care, bringing them back into the facility would potentially increase their exposure unnecessarily. If their risk of HIV acquisition is high (have a partner living with HIV or unknown serostatus, multiple partners, or a sexually transmitted infection), then the benefit may outweigh the risk. But for women who are not at high risk of HIV acquisition, the risk of exposure to coronavirus would outweigh the benefit.

Our study of PrEP in pregnancy halted enrolment of pregnant women onto PrEP in light of the potential risk of coronavirus exposure to our research staff and study participants, but we are continuing with our retention and prescription visits. We ensure that clinical staff have personal protective equipment (PPE) and adequate physical distancing. We also use system and offer research study visits outside of the busy health facility. Adequate consultation with staff, health facility and community also important. However, fear surrounding the health care facility, being outside of one's home with the military presence, and transmission risk, may keep women inside rather than attending her antenatal, postnatal and PrEP study visits.

\section{Benefits of Continuation: Enabling Pregnant and Breastfeeding Women to Prevent HIV Acquisition During High Risk Period of Societal Lockdown}

HIV incidence is high during pregnancy and breastfeeding [2] with HIV acquisition risk more than doubling during pregnancy and the postpartum period compared to when women are not pregnant [14]. A recent meta-analysis demonstrated that the mean HIV incidence rate among pregnant and breastfeeding women was 3.6 per 100 person-years (95\% prediction interval: 1.2-11.1) [1]. In Cape Town, a study demonstrated that HIV incidence was $1.86 / 100$ person-years in postpartum period (95\% confidence interval 0.88-3.89) with a very high vertical transmission to $28 \%$ infants [15]. HIV acquisition during pregnancy and postpartum translates to a substantial cumulative period of risk over the course of women's lives in sub-Saharan African regions where both fertility and HIV prevalence are high [2]. Additionally, acute maternal HIV infection during pregnancy and breastfeeding threatens maternal health and increases the risk of vertical transmission accounting for an estimated $30 \%$ of new HIV infections in some settings $[10,16,17]$. In South Africa, an estimated 76,000 (95\% CI 64,000-90,000) new cases of vertical transmission are expected over the 2020-2030 period [18]. By providing PrEP in pregnancy, we estimate a significant reduction of over $41 \%$ with PrEP uptake in $80 \%$ of pregnant women, or $13 \%$ if one-third of high-risk women take PrEP [18]. Coronavirus could cause appreciable morbidity in pregnant women and infants, requiring hospitalization which could have lifelong implications for chronic lung disease, bronchiectasis - though there is limited evidence of this risk in pregnant women $[19,20]$.

In a recent systematic review done by our group, we identified 14 completed $(n=5)$ and ongoing/planned $(n=9)$ studies that are evaluating maternal and/or infant outcomes following PrEP exposure during pregnancy or breastfeeding. Nine ongoing studies, to be completed by 2022 , will provide data on $>6200$ additional PrEP-exposed pregnancies and assess perinatal, infant growth, and bone health outcomes, expanding by sixfold the data on PrEP safety in pregnancy [21]. Coronavirus will delay these important results that are set to support guideline development and national roll out of PrEP in pregnancy programs.

Prior research has shown that during emergencies and disasters like coronavirus, in which people are told to stay home, sexual activity will continue and increase. Consequently, the risks of intimate partner violence, rape and HIV acquisition will also rise $[12,13]$. The South African government reported over 87,000 cases of gender based violence reported across the country in the first week of the coronavirus lockdown [20]. We have never lived through an epidemic like this in which people are forced to stay home, oftentimes in very tight conditions. In a South African township, living conditions are extremely crowded. Socializing is unavoidable. Many don't have water and need to stand in queues to get water or food. The major components recommended-social distancing and hygiene-are extremely difficult to implement in much of Africa. In this new world of societal lockdown, pregnant and breastfeeding women who were once at low risk of HIV acquisition could now be 
at high risk. In a migratory society, male partners are now home and are no longer migrating, increasing sexual exposure in a population where condomless sex is high and many women have limited power to effectively negotiate condom use [22, 23]. Finally, HIV is a lifelong virus that affects the most vulnerable in our society, regardless of age. Though there is effective treatment of HIV, there is no vaccine or cure for HIV.

\section{Conclusion}

Given the evidence and our experience, we argue that the benefits outweighs the risks in pregnant women and advocate for continued PrEP provision and HIV risk reduction counselling in HIV-uninfected pregnant and breastfeeding women at high-risk of HIV acquisition in South Africa.

Acknowledgements DJD received funding from the National Institute of Health and Fogarty International Center (K01TW011187). TC and LM received funding from the National Institute of Mental Health (R01MH116771)

\section{References}

1. Graybill LA, Kasaro M, Freeborn K, et al. Incident HIV among pregnant and breast-feeding women in sub-Saharan Africa: a systematic review and meta-analysis. AIDS (London, England). 2020;34(5):761-76.

2. Drake AL, Wagner A, Richardson B, John-Stewart G. Incident HIV during pregnancy and postpartum and risk of mother-to-child HIV transmission: a systematic review and meta-analysis. PLoS Med. 2014;11(2):e1001608.

3. Chutel L, Dahir AL. With most coronavirus cases in Africa, South Africa Locks Down. New York Times; 2020.

4. South Africa Department of Health. Corona Virus (COVID-19) - updated. 2020. Accessed 29 Mar 2020.

5. Fauci AS, Lane HC, Redfield RR. Covid-19-Navigating the uncharted. New Engl J Med. 2020;382(13):1268-9.

6. UNAIDS. UNAIDS Data 2019. Geneva, Switzerland; 2019.

7. Council HSR. Incidence, Behaviour and Communication Survey, "South African National Prevalence, Incidence, Behaviour and Communication Survey". https://www.hsrcacza/en/media-briefs/ hiv-aids-stis-and-tb/sabssm-launch-2018v2; 2018.

8. South Africa Department of Health. Guidelines for the provision of pre-exposureprophylaxis (PrEP) to persons at substantial risk of HIV infection"; 2019.

9. Stats South Africa. Mid-year population estimates, 2018. 2019. Accessed 6 Apr 2020

10. UNAIDS. Start Free Stay Free AIDS Free. Geneva, Switzerland; 2019.
11. University of Cape Town Human Research Ethics Committee. COVID-19: Faculty of health sciences human research ethics committee recommendations for research studies and research sites involving humans. Cape Town, South Africa: UCT; March 17, 2020. p. 2.

12. Muluneh MD, Stulz V, Francis L, Agho K. Gender based violence against women in sub-Saharan Africa: a systematic review and meta-analysis of cross-sectional studies. Int J Environ Res Public Health. 2020;17(3):903.

13. South African Government News Agency. Over 2000 arrests for non-compliance with COVID-19 rules. https://www.sanews.gov. $\mathrm{za} / .2020$.

14. Thomson KA, Hughes J, Baeten JM, et al. Increased risk of female HIV-1 acquisition throughout pregnancy and postpartum: a prospective per-coital act analysis among women with HIV-1 infected partners. J Infect Dis. 2018;218:16-25.

15. le Roux SM, Abrams EJ, Nguyen KK, Myer L. HIV incidence during breastfeeding and mother-to-child transmission in Cape Town, South Africa. AIDS (London, England). 2019;33(8):1399-401.

16. Johnson LF, Stinson K, Newell ML, et al. The contribution of maternal HIV seroconversion during late pregnancy and breastfeeding to mother-to-child transmission of HIV. J Acquir Immune Defic Syndr. 2012;59(4):417-25.

17. Dinh TH, Delaney KP, Goga A, et al. Impact of maternal HIV seroconversion during pregnancy on early mother to child transmission of HIV (MTCT) measured at 4-8 weeks postpartum in South Africa 2011-2012: a national population-based evaluation. PLoS ONE. 2015;10(5):e0125525.

18. Joseph Davey DL, Bekker LG, Gomba Y, Coates T, Myer L, Johnson LF. Modelling the potential impact of providing preexposure prophylaxis in pregnant and breastfeeding women in South Africa. AIDS (London, England). 2019;33(8):1391-5.

19. Chen S, Liao E, Shao Y. Clinical analysis of pregnant women with 2019 novel coronavirus pneumonia. J Med Virol. 2020. https:// doi.org/10.1002/jmv.25789.

20. Chen $\mathrm{T}, \mathrm{Wu} \mathrm{D}$, Chen $\mathrm{H}$, et al. Clinical characteristics of 113 deceased patients with coronavirus disease 2019: retrospective study. BMJ. 2020;368:m1091.

21. Joseph Davey DL, Pintye J, Baeten JM, et al. Emerging evidence from a systematic review of safety of pre-exposure prophylaxis for pregnant and postpartum women: where are we now and where are we heading? J Int AIDS Soc. 2020;23(1):e25426.

22. Joseph Davey D, Peters RPH, Kojima N, et al. Sexual behaviors of human immunodeficiency virus-infected pregnant women and factors associated with sexually transmitted infection in South Africa. Sex Transm Dis. 2018;45(11):754-61.

23. Joseph Davey D, Farley E, Gomba Y, Coates T, Myer L. Sexual risk during pregnancy and postpartum periods among HIVinfected and -uninfected South African women: implications for primary and secondary HIV prevention interventions. PLoS ONE. 2018;13(3):e0192982.

Publisher's Note Springer Nature remains neutral with regard to jurisdictional claims in published maps and institutional affiliations. 